\title{
Handling Non-functional Requirements in Model-Driven Development: An Ongoing Industrial Survey
}

\author{
David Ameller*, Xavier Franch*, Cristina Gómez*, \\ Joao Araujo ${ }^{\dagger}$, Richard Berntsson Svensson ${ }^{\ddagger}$, Stefan Biffl ${ }^{\S}$, Jordi Cabot ${ }^{\dagger}$, Vittorio Cortellessa**, Maya Daneva ${ }^{\dagger \dagger}$, \\ Daniel Méndez Fernández ${ }^{\dagger \ddagger}$, Ana Moreira ${ }^{\dagger}$, Henry Muccini**, Antonio Vallecillo ${ }^{x}$, Manuel Wimmer ${ }^{\S}$, \\ Vasco Amaral ${ }^{\dagger}$, Hugo Brunelière ${ }^{\mathrm{xi}}$, Loli Burgueño ${ }^{x}$, Miguel Goulão ${ }^{\dagger}$, Bernhard Schätz ${ }^{\text {xii }}$ and Sabine Teufl ${ }^{\text {xii }}$ \\ *Universitat Politècnica de Catalunya, Spain. \{dameller, franch, cristina\}@essi.upc.edu \\ ${ }^{\dagger}$ NOVA LINCS, Universidade Nova de Lisboa, Portugal. \{p191, amm, vasco.amaral, mgoul $\} @$ fct.unl.pt

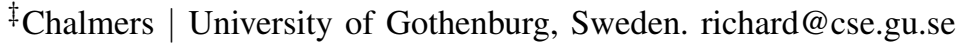 \\ $\S$ Vienna University of Technology, Austria. Stefan.Biffl@tuwien.ac.at, Wimmer@big.tuwien.ac.at \\ IICREA - UOC, Spain. jordi.cabot@icrea.cat \\ **University of L'Aquila, Italy. \{vittorio.cortellessa, henry.muccini\}@ univaq.it \\ ${ }^{\dagger}$ University of Twente, The Netherlands. M.Daneva@utwente.nl \\ $\ddagger_{\ddagger}$ Technische Universität München, Germany. mendezfe@in.tum.de \\ ${ }^{x}$ Universidad de Málaga, Spain. \{av, loli\}@1cc.uma.es \\ ${ }^{x i}$ AtlanMod Team (Inria, Mines Nantes \& LINA), France. hugo.bruneliere@inria.fr \\ ${ }^{x i i}$ Fortiss GmbH, Germany. \{schaetz, teufl $\} @$ fortiss.org
}

\begin{abstract}
Model-Driven Development (MDD) is no longer a novel development paradigm. It has become mature from a research perspective and recent studies show its adoption in industry. Still, some issues remain a challenge. Among them, we are interested in the treatment of non-functional requirements (NFRs) in MDD processes. Very few MDD approaches have been reported to deal with NFRs (and they do it in a limited way). However, it is clear that NFRs need to be considered somehow in the final product of the MDD process. To better understand how NFRs are integrated into the existing MDD approaches, we have initiated the NFR4MDD project, a multi-national empirical study, based on interviews with companies working on MDD projects. Our project aims at surveying the state of the practice for this topic. In this paper, we summarize our research protocol and present the current status of our study. The discussion will focus on the peculiarities of our study's context and organization involving about 20 researchers from 8 European countries.
\end{abstract}

Index Terms-Non-Functional Requirements, Model-Driven Development, Quality Requirements, NFR, MDD, Empirical Study, Survey, Semi-Structured Interviews.

\section{INTRODUCTION}

Model-Driven Development (MDD) is a development paradigm, where models play a central role [1], [2]. "Modeldriven development is simply the notion that we can construct a model of a system that we can then transform into the real thing” [2]. One of the benefits of using MDD is the higher abstraction level by providing platform independence. Other related concepts are Model-Driven Architecture (MDA) [3], the OMG perspective of MDD; and Model-Driven Engineering (MDE) [4], which includes other software engineering activities in addition to development. In this paper, we will focus on MDD approaches for software production.
Non-Functional Requirements (NFRs) are one of the main research targets in the Requirements Engineering community [5] and their industrial impact has been documented in many empirical studies (e.g., [6], [7], [8], [9]). There are many definitions for the concept of NFR [5]. For example, they have been defined as: “(...) global requirements on [the system] development or operational cost, performance, reliability, maintainability, portability, robustness, and the like." [10]. However there is no common agreement on the concrete meaning of the term NFR [11].

Dealing with NFRs is a major challenge in software development. The lack of integration of NFRs with functional requirements can result in increased time-to-market and project cost [5], [12]. This holds also for MDD. Being supported or not by MDD approaches, practitioners have to deal with NFRs in one way or another.

Ameller et al. [13] presented at RE'10 a vision paper on the impact of NFRs in MDD processes and foresaw different ways to handle them. The authors pointed out the lack of support of NFRs in MDD (based on a literature review) and hypothesized that this situation is probably even more dramatic in the industrial practice. This topic was also subject of a keynote ${ }^{1}$, given at the MoDRE@RE'14 workshop, where some industrial attendees and academics with industrial experience reinforced this hypothesis through their questions and opinions. Moreover, recent empirical studies that report the adoption of MDD in industry [14], [15], [16], [17] do not provide insights on how practitioners deal with NFRs.

\footnotetext{
${ }^{1}$ www.slideshare.net/gessiupc/mo-dre-2014-keynote
} 
TABLE I

RESEARCH QUESTIONS

\begin{tabular}{|l|l|}
\hline RQ1 & In which context is MDD adopted by companies? \\
\hline RQ1.1 & What factors motivate the adoption of MDD? \\
\hline RQ1.2 & Which types of NFRs are linked to these motivating factors? \\
\hline RQ1.3 & To what extent are NFRs relevant for those projects that adopt MDD? \\
\hline RQ2 & To what extent do MDD approaches adopted by companies support NFRs? \\
\hline RQ2.1 & Which types of NFRs are supported by the adopted MDD approaches? \\
\hline RQ2.2 & Which characteristics do these NFRs exhibit? \\
\hline RQ2.3 & Which notations and tools are used for the supported types of NFRs? \\
\hline RQ2.4 & At which stages of the adopted MDD approach are these NFRs handled? \\
\hline RQ3 & How do companies deal with NFRs when the adopted MDD approach does not support them? \\
\hline RQ3.1 & How are MDD approaches customized to take into account the previously unsupported types of NFRs? \\
\hline RQ3.2 & How do companies deal with an NFR which is not supported by MDD? \\
\hline RQ3.3 & To what extent do the drawbacks of dealing with unsupported types of NFRs compensated by the benefits of adopting MDD? \\
\hline
\end{tabular}

Further investigating this hypothesis is the main motivation of our work. In this paper, we present the ongoing NFR4MDD project, a multinational (Europe-wide) empirical study on the industrial practices in MDD, focusing on how companies handle NFRs when using MDD. The study involves about 20 researchers from 8 countries and is expected to involve around 30 companies whose experiences and perceptions will be surveyed using semi-structured interviews.

The rest of the paper is divided into the following sections: Section II, the objective and the research questions of this work; Section III, the context in which this research is being held; Section IV, the working plan and the research methodology; and finally Section V, the conclusions.

\section{ObJective of the StUdy}

The main goal of the NFR4MDD project is to obtain a detailed view on the way that companies handle NFRs when they use MDD. Based on this goal, we define the research questions (RQs) shown in Table I.

The first RQ (RQ1) shall provide an overview of the context in which MDD approaches are used by European companies. In particular, we are interested in identifying the factors (e.g., speed up the development process, improve the reusability, better documentation) that motivate the adoption of MDD as the most suitable option (RQ1.1). Specifically, we want to search for links between these factors and specific types of NFRs (RQ1.2). Since our working hypothesis is that most MDD approaches do not handle any type of NFRs, with RQ1.3 we want to know if MDD is adopted in projects where the NFRs are less important than functional requirements, or whether relevant NFRs are already satisfied by the technologies or the infrastructure used. While RQ1.1 shall allow us to compare our findings with other surveys on MDD in practice (see Section I), RQ1.2 and RQ1.3 aim at characterizing the participating companies with regard the topic of study.

The second RQ (RQ2) is focused on understanding how NFRs are supported by the MDD approaches currently adopted by the participating companies. First, we want to know if the MDD approaches adopted by the companies tend to support any specific NFR (RQ2.1). For the supported NFRs, we want to determine: a) their characteristics, for example, how near or far the NFRs are from the solution, the granularity of their specification, etc. (RQ2.2); b) how are they represented in the models, for example, the languages or extensions used (RQ2.3). Also, we want to map the supported NFRs in the adopted MDD approaches, in particular into the stages at which the NFRs are handled (RQ2.4). As part of our study, we plan to compare the results of the survey to previously published findings.

Even if some types of NFRs might be supported by the MDD approaches adopted by companies, in most cases we expect to find only limited support [13]. For this reason, we aim with RQ3 at understanding the strategies used by the companies when they have to deal with those types of NFRs that are unsupported by the MDD approaches adopted in their context. Ameller et al. [13] envisaged two possible strategies: the first one is to adapt the MDD process so that it can account for the unsupported types of NFRs (RQ3.1) and the second one is to manually adapt the resulting artifact of the MDD process in order to satisfy the unsupported NFRs (RQ3.2). However, we expect that companies may well use other strategies, for example, postpone the NFRs for future releases, or simply discard the NFRs (RQ3.2). Finally, since all of these situations can affect the development, we want to better understand their impact from the point of view of the participating companies (RQ3.3), i.e., we want to determine if bearing the drawbacks due to the loose integration of NFRs into the MDD process is compensated by the benefits provided by MDD approaches.

\section{Context of The Study}

The NFR4MDD project was initiated by the Universitat Politècnica de Catalunya (UPC) team after the keynote at MoDRE@RE'14 mentioned in Section I. The collective observation that the challenge remains open and the interest 


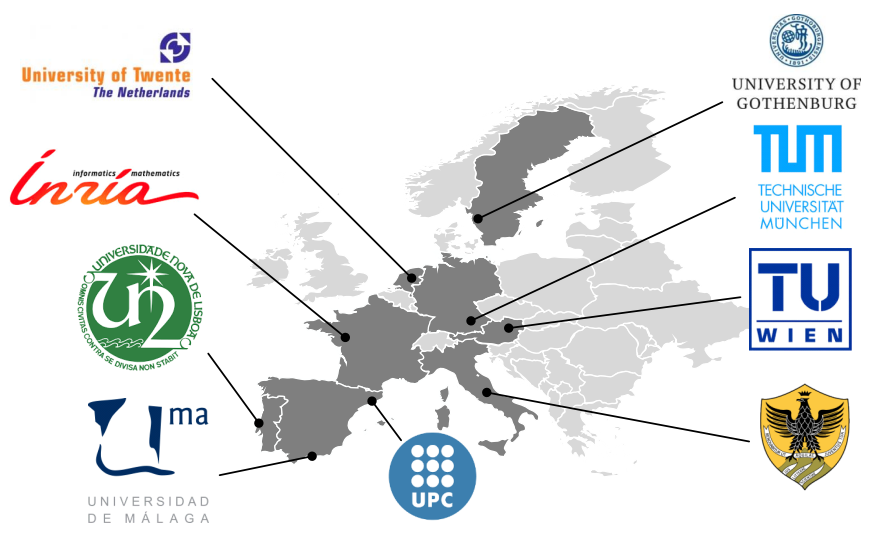

Fig. 1. Participant countries.

expressed by the community were the triggers for the study described in this paper.

The collaborative nature of the survey was also decided from the very beginning. Not just the difficulty of getting enough subjects by only one research group was considered, but in fact the main driver for this decision was to aim at the best possible quality of the project and its outcome by involving experienced researchers of the two main areas of the study (NFRs and MDD). A subsequent decision was that UPC would act as a research coordinator. The criteria that UPC used to select participating researchers were:

- European research groups. We invited only one research group per country, thus ensuring representation of countries is well balanced (see Fig. 1). Differences emerging from the characteristics of a particular country may eventually be investigated.

- The researchers should have demonstrated expertise in at least one of the main areas of the study, MDD or NFRs.

- The researchers should have profound expertise in empirical research methods, ideally in qualitative research. In particular, membership to the ISERN network ${ }^{2}$ was welcome.

The invited researchers received a formal invitation letter ${ }^{3}$ composed of the following parts: general presentation of the project, research questions, list of responsibilities, and the initial work plan. In general, the response was quite positive, especially considering that NFR4MDD is not a funded project.

With regards to the responsibilities, the coordinators of the project will lead most of the tasks, while the participating researchers will collaborate in the: review of the protocol, search for interviewees in companies using MDD, execution of interviews, feedback on data analysis, and collaboration in reporting (e.g., writing papers).

\section{A. Management Issues}

Coordinators had to deal with several management issues. For instance, to discuss the preliminary versions of documents

\footnotetext{
${ }^{2}$ isern.iese.de

${ }^{3}$ www.essi.upc.edu/ gessi/papers/RE15-letter.pdf
}

we opted to use collaborative space that allows several researchers working at the same time on the same document without conflicts. For the consolidated versions of the documents and the research material (e.g., interview transcriptions, data analysis) we use a shared repository with easy-to-use storage capabilities. We also created a mailing list to be used as the main communication channel of the project.

Not surprisingly for such a collaborative endeavor, several circumstances started to arise from the very beginning, e.g.:

- Initially, it was planned to have two researchers per country, but some countries kindly required to have more than two researchers, therefore it was decided to exceptionally allow to exceed the original numbers.

- Even in a short space of time, some researchers started to change affiliation and even country. This will not be considered a problem as far as the interviews are conducted in the originally planned country.

- We wanted to define as soon as possible the author order in the artifacts produced in the project (in particular, research papers). Authorship rules (already applied in the paper at hand) are: first, coordinators; next, national representatives; last, supporting researchers. Alphabetical order is proposed in every category.

- Some of the research groups have expressed concerns about the need to record and transcribe the interviews (e.g., national privacy regulations). Here we opted to go case-by-case in order to find the best solution that minimizes the impact on the quality of the study.

- The language used in the interviews was also a matter of discussion. The final decision was to use at every interview the most appropriate language, with the only restriction to provide the final transcription in English.

\section{B. Open Discussions}

More interestingly, we started some discussions about the contents of our empirical study, e.g.:

- We are currently deciding whether we use a common NFRs taxonomy or the taxonomy that better fits the company domain in each case and we consolidate terminology during data analysis. One reason is that we expect a varying understanding of the notion of NFRs for the various industrial sectors and application domains covered by our study ("there is not any formal definition or complete list of nonfunctional requirements" [10]).

- We are currently discussing the various context- and domain-specific interpretations of MDD and we are considering to initiate the interviews by letting the participants define the notion of MDD for their context first.

- We initially proposed that each country representative would select their participants, but as some concerns about the diversity arose, we decided to make the selection at a global level, once all the countries have proposed their own candidates. 


\section{Planning of the Study}

Among the variety of types of empirical studies (e.g., surveys, case studies, experiments), this study will be a qualitative survey. Surveys (e.g., interviews, online questionnaires) are a common way for collecting qualitative and quantitative information [18]. They provide a snapshot of the current state of the studied topic by collecting information to describe, compare, or explain knowledge, attitudes and behavior [18]. Surveys aim at understanding a population from which a sample will be drawn.

This survey will include descriptive and exploratory intentions. Descriptive surveys are conducted to enable assertions about some population. They are designed to measure what occurred, rather than why. Exploratory surveys are used as a pre-study to a more thorough investigation.

We followed Ciolkowski et al.'s guidelines [19] to define a protocol for this study. In these guidelines the following steps are defined (iterations are not represented):

1) Survey definition. Objective and research questions.

2) Survey design. Planning the key elements of the study, including population, sample, variables, data collection and analysis, and mitigation of threats to validity.

3) Survey implementation. Make the survey executable.

4) Survey execution. Data collection and processing.

5) Survey analysis. Analysis and interpretation.

6) Survey packaging. Report the results of the study.

\section{A. Survey Steps}

Our survey definition (Step 1) is stated in Section II. In this section we focus on the survey design (Step 2) and we outline the remaining steps to provide a full overview of the process and the planned work.

Following the guidelines in [19], the survey design should consider: the target population and the survey sample; the variables of the survey; the approach for data collection; the questionnaire design; the approaches for data analysis; and the threats to validity.

Population. Our population is the software companies. We do not restrict our population with regard to the company size or application domain, but we require experience using MDD in software projects. We deliberately avoided a more restrictive population in order to facilitate the selection of candidates and to avoid a narrow view on the topic of study. However, we require that the representative of each participating company has the adequate background in MDD (i.e., s/he has participated, at least, in one project that applied MDD in the company).

Sampling. The sampling method used in this study is nonprobability sampling. As explained in Section III, our selection of candidates is made up by the national representatives using their known industrial contacts. Taking into account the geographical distribution of the participants, we consider that our sampling method is a good approach to obtain the sample of the target population. Since we do not have a sufficient theoretical basis to make estimates on the target population, we will limit our findings to the obtained sample rather than inferences over the population.
Variables. Next we defined the list of variables linked to the research questions and the questions in the questionnaire. These variables form the basis of the survey analysis (Step 5). It is worth mentioning that even if we made an effort to determine most of the variables that will appear in the survey, some new ones may appear during the survey execution in Step 4 (e.g., some recurrent pattern during the content analysis).

Data collection. The data collection instrument, as already mentioned, will be face-to-face interviews, which gives us the possibility to get the participant answer our questions without skipping any question (providing clarifications when necessary). Also, it is possible for the interviewer to observe and ask questions to the participants to extend their responses (e.g., to understand the reasoning of the participant, or to go deeper into the details).

Questionnaire design. We followed the writing recommendations given by Dillman et al. [20] to reduce the common mistakes when designing a questionnaire (e.g., keep positive and negative sides in the questions; questions polarized to one side can bias the responses). We also made several internal iterations to improve the questionnaire, and we planned internal and real-case pilots before proceeding to the survey execution.

Data analysis. The obtained data will be analyzed using basic descriptive analysis and content analysis. For the basic descriptive analysis, we will use frequency analysis and comparison of different variables. The use of statistical correlation analysis may not be feasible due to the expected sample size. For the content analysis, we will identify categories using coding techniques, and we will use qualitative analysis techniques (which often work well with small samples) such as summarizing, explaining, and structuring [21].

Threats to validity. We identified the most relevant threats of internal, external, conclusion, and construct validity as defined in the book by Wohlin et al. [22]. The next subsection details the most relevant threats to the planned survey.

\section{B. Threats to Validity}

1) Internal Validity: Understandability problems. Some questions may be understood differently of what they are intended for resulting in lower quality answers, or even not valid answers. As mitigation the questionnaire was designed following the indications given by Dillman et al. [20] and will be piloted in several iterations to ensure its understandability.

Language difficulties. The language used during the interviews will be chosen by the participant, therefore, in some cases, the transcription of the interview will be translated to English. During the translation, some information may be lost or incorrectly translated, especially for technical terms. As mitigation, the translation will be verified by the local researchers who had participated in the interview and know the technical terms of the research topic.

Insufficient knowledge of the participant. Having a wrong profile participating in the survey may result in lower quality answers, or the inability of the participant to provide an answer to some questions. To mitigate this problem, the selected participants should have experience using MDD; more 


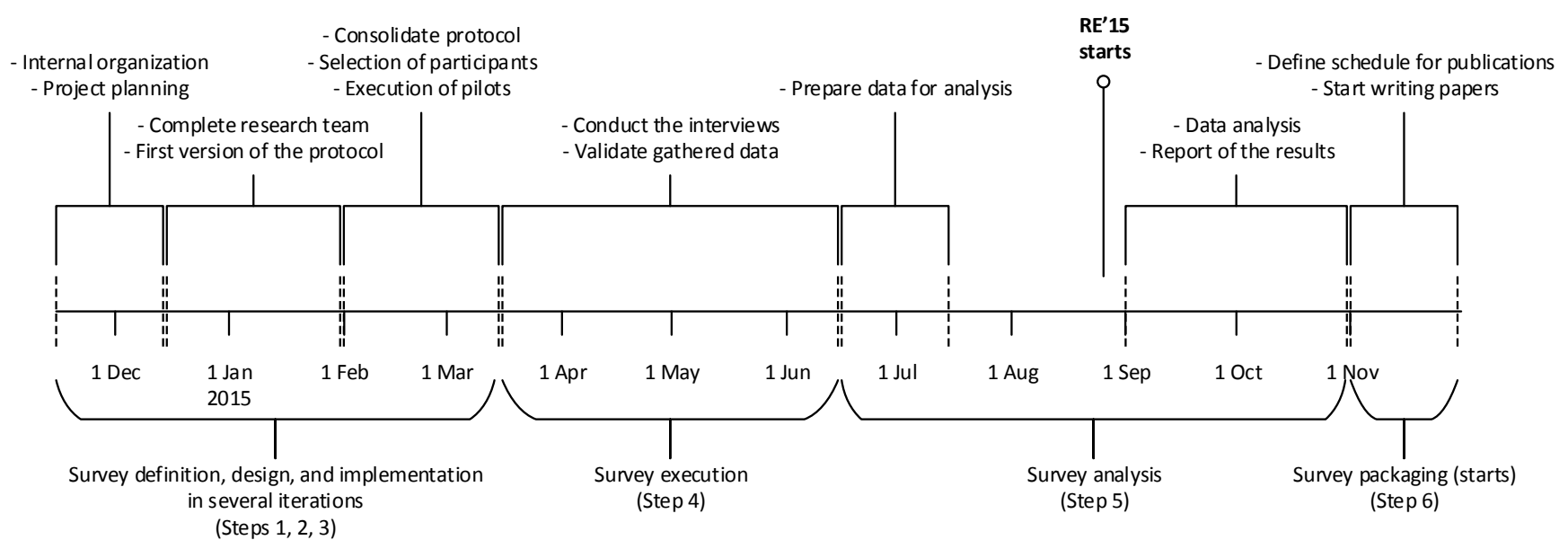

Fig. 2. Timeline of the work plan.

concretely, they should have participated as software engineers in at least one MDD project that has successfully finished in their current company.

Untruthful answers. Participants may be reluctant to provide sincere responses when they have to explain negative aspects about their work and their company. In order to minimize this situation, when the survey is executed, the interviewers will inform the participants that the data collected will be used anonymously and that the purpose of the interview is not to evaluate them or their company.

2) External Validity: As it is the usual case in interviewbased surveys, the sample size and the sampling technique used do not provide the statistical basis to generalize the results to the target population. The results should yield, however, an initial theory which can be used to steer future research.

3) Conclusion Validity: Interviewers' bias. Due to the context of this study, the interviews will be executed by several different interviewers, therefore there is a risk that they conduct the interview in different ways (e.g., making more emphasis on some parts than others). As countermeasure we are creating documentation for the execution: a guide for the execution with the specific steps that need to be performed before, during and after the interviews, and a questionnaire populated with specific instructions for the interviewers. In addition, we will hold a plenary meeting before starting the execution phase to explain and clarify all the aspects related to the interviews execution.

Replicability of this study. Once the study is finalized, the protocol, and all the related material used to perform this study will be made available under $\mathrm{CC}-\mathrm{BY}^{4}$ license. The open character of our project will support researchers and practitioners to replicate this study, and, in the long run, to generalize and verify the results.

4) Construct Validity: The underlying methodology is not robust. The protocol may be incomplete, or provide insufficient details for the success of the project. To guarantee

\footnotetext{
${ }^{4}$ creativecommons.org/licenses/by/4.0
}

a solid foundation, we have followed Ciolkowski et al.'s guidelines [19]. These guidelines cover the most important aspects to be considered when performing a survey. As result, we defined a detailed protocol ${ }^{5}$.

The questionnaire ${ }^{6}$ used to drive the semi-structured interview is not well linked to the research questions. Missing important data during the interviews may lead to the impossibility to provide an answer to some of the research questions. To verify the suitability of the questionnaire, we have related each question in the questionnaire with one or more variables (to be used for the analysis), and these variables with the research questions (one variable may provide insightful information for more than one research question). We are currently working on a conceptual model that clarifies the relationships between the variables (e.g., context, input and output factors of the MDD process), which we will use as the foundation for our research questions. The model will be available in the next iteration of the protocol.

\section{Work Plan}

Due to the large number of researchers involved in this study, it was deemed critical to put in place a well-defined work plan since the very beginning. The work plan and its timeline is depicted in Fig. 2. It shows the tasks that have been and will be performed.

The current status (at the time of submitting this paper) is a complete survey definition and design (Steps 1 and 2), which may still be subject of minor modifications. For the survey implementation (Step 3), our first version of the questionnaire is almost ready but the pilots are to be executed. Steps 4 and 5 are not initiated yet. This paper can be seen as part of the reporting activities of the survey (which belong to Step 6). As part of the survey packaging we envision several publications at conferences and in journals, including RE' 16.

\footnotetext{
${ }^{5}$ www.essi.upc.edu/ $\sim$ gessi/papers/RE15-protocol.pdf

${ }^{6}$ www.essi.upc.edu/ gessi/papers/RE15-questionnaire.pdf
} 


\section{CONCLUSION}

In this paper, we have reported the planning and current status of an empirical study about the management of NFRs in MDD processes in industry. We have explained the objective and the research questions, the context, the methodology used, and the planning of the tasks remaining. We have explained the difficulties that we have encountered during the organization of this project together with our solutions. We also have discussed the threats to the validity of the survey and selected mitigation actions for these threats.

The RE Next call for papers has provided us with a great opportunity for the study. First, given the current preliminary stage, we expect to get relevant feedback from the reviewers for improving our protocol in a timely manner. Should the paper be accepted, the presentation at the conference itself would provide an excellent opportunity to share our first impressions on the ongoing analysis and raise awareness of the study. The writing of the paper itself has also represented a kind of proof of concept for the full study in terms of collaborative work. In this respect, this paper has served as a motivating instrument to activate the participation of all researchers involved. We also consider that this paper provides a valuable experience in organizing such an Europe-wide kind of study which could help other researchers organizing similar collaborative projects.

Once this study is completed, our intention is to extend it to worldwide scale. We are open for future iterations with participants from all the continents. We think that the RE conference will be a great venue to foster such participation.

\section{ACKNOWLEDGMENT}

Several projects and grants have supported our work: Secretaria d'Universitats i Recerca de Catalunya; TIN201344641-P; TIN2011-23795; FCT/MEC NOVA LINCS PEst UID/CEC/04516/ 2013; the Christian Doppler Forschungsgesellschaft and the BMWFJ, Austria.

\section{REFERENCES}

[1] C. Atkinson and T. Kühne, "Model-Driven Development: a metamodeling foundation," IEEE Software, vol. 20, no. 5, pp. 36-41, Sep. 2003.

[2] S. J. Mellor, A. N. Clark, and T. Futagami, "Guest Editors' Introduction: Model-Driven Development," IEEE Software, vol. 20, pp. 14-18, 2003.

[3] J. Miller and J. Mukerji, "MDA Guide Version 1.0.1," Object Management Group (OMG), Tech. Rep., 2003

[4] D. C. Schmidt, "Guest Editor's Introduction: Model-Driven Engineering," Computer, vol. 39, no. 2, pp. 25-31, Feb. 2006.

[5] L. Chung and J. C. S. do Prado Leite, Conceptual Modeling: Foundations and Applications. Springer, 2009, ch. On Non-Functional Requirements in Software Engineering, pp. 363-379.
[6] D. Ameller, C. Ayala, J. Cabot, and X. Franch, "How do Software Architects Consider Non-functional Requirements: An Exploratory Study," in 20th IEEE International Requirements Engineering Conference (RE), 2012, pp. 41-50.

[7] D. Ameller, M. Galster, P. Avgeriou, and X. Franch, "A survey on quality attributes in service-based systems," Software Quality Journal, 2015.

[8] R. Berntsson Svensson, T. Gorschek, B. Regnell, R. Torkar, A. Shahrokni, and R. Feldt, "Quality Requirements in Industrial Practice: An Extended Interview Study at Eleven Companies," IEEE Trans. Softw. Eng., vol. 38, no. 4, pp. 923-935, Jul. 2012.

[9] M. Daneva, S. Marczak, and A. Herrmann, "Engineering of Quality Requirements As Perceived by Near-shore Development Centers' Architects in Eastern Europe: The Hole in the Whole," in Proceedings of the 8th ACM/IEEE International Symposium on Empirical Software Engineering and Measurement, 2014.

[10] J. Mylopoulos, L. Chung, and B. A. Nixon, "Representing and Using Nonfunctional Requirements: A Process-Oriented Approach," IEEE Trans. Softw. Eng., vol. 18, no. 6, pp. 483-497, Jun. 1992.

[11] M. Glinz, "On Non-Functional Requirements," in 15th IEEE International Requirements Engineering Conference (RE'07), 2007, pp. 21-26.

[12] L. Chung, B. A. Nixon, E. Yu, and J. Mylopoulos, Non-functional requirements in software engineering. Kluwer Academic, 2000.

[13] D. Ameller, X. Franch, and J. Cabot, "Dealing with Non-Functional Requirements in Model-Driven Development," in 18th IEEE International Requirements Engineering Conference (RE), 2010, pp. 189-198.

[14] J. Hutchinson, J. Whittle, and M. Rouncefield, "Model-Driven Engineering Practices in Industry: Social, Organizational and Managerial Factors that lead to Success or Failure," Science of Computer Programming, vol. B, no. 89, pp. 144-161, September 2014.

[15] P. Mohagheghi, W. Gilani, A. Stefanescu, and M. Fernandez, "An empirical study of the state of the practice and acceptance of model-driven engineering in four industrial cases," Empirical Software Engineering, vol. 18, no. 1, pp. 89-116, 2013.

[16] L. T. W. Agner, I. W. Soares, P. C. Stadzisz, and J. M. Simão, “A Brazilian survey on UML and model-driven practices for embedded software development," Journal of Systems and Software, vol. 86, no. 4, pp. 997-1005, 2013.

[17] M. Torchiano, F. Tomassetti, F. Ricca, A. Tiso, and G. Reggio, "Relevance, benefits, and problems of software modelling and model driven techniques: A survey in the Italian industry," Journal of Systems and Software, vol. 86, no. 8, pp. 2110-2126, 2013.

[18] C. Wohlin, M. Höst, and K. Henningsson, "Empirical Research Methods in Software Engineering," in Empirical Methods and Studies in Software Engineering, ser. Lecture Notes in Computer Science, R. Conradi and A. Wang, Eds. Springer Berlin Heidelberg, 2003, vol. 2765, pp. 7-23.

[19] M. Ciolkowski, O. Laitenberger, S. Vegas, and S. Biffl, "Practical Experiences in the Design and Conduct of Surveys in Empirical Software Engineering," in Empirical Methods and Studies in Software Engineering, ser. Lecture Notes in Computer Science, R. Conradi and A. Wang, Eds. Springer Berlin Heidelberg, 2003, vol. 2765, pp. 104-128.

[20] D. A. Dillman, J. D. Smyth, and L. M. Christian, Internet, Phone, Mail, and Mixed-Mode Surveys: The Tailored Design Method, 4th ed. Wiley Publishing, 2014.

[21] P. Mayring, Qualitative Content Analysis. Theoretical Foundation, Basic Procedures and Software Solution. Social Science Open Access Repository, 2014. [Online]. Available: http://nbn-resolving.de/urn:nbn: de:0168-ssoar-395173

[22] C. Wohlin, P. Runeson, M. Hst, M. C. Ohlsson, B. Regnell, and A. Wesslén, Experimentation in Software Engineering. Springer, 2012. 